\title{
DISTRIBUTIONS OF OCCUPATION TIMES OF BROWNIAN MOTION WITH DRIFT
}

\author{
ANDREAS PECHTL \\ Center of Asset Pricing and Financial Products Development \\ Deutsche Genossenschaftsbank Frankfurt am Main \\ Am Platz der Republik, D-60325 \\ Frankfurt am Main, Germany
}

\begin{abstract}
The purpose of this paper is to present a survey of recent developments concerning the distributions of occupation times of Brownian motion and their applications in mathematical finance. The main result is a closed form version for Akahori's generalized arc-sine law which can be exploited for pricing some innovative types of options in the Black \& Scholes model. Moreover a straightforward proof for Dassios' representation of the $\alpha$-quantile of Brownian motion with drift shall be provided.
\end{abstract}

Keywords: Brownian motion with drift, occupation times, Black \& Scholes model, quantile options.

\section{INTRODUCTION}

Problems of pricing derivative securities in the traditional Black \& Scholes framework are often closely connected to the knowledge of distributions induced by application of measurable functionals to Brownian motion. Familiar types of measurable functionals such as passage times and maximum or minimum functionals were already considered in early investigations of Brownian motion and - from a stochastic point of view - are responsible for popular exotic option constructions like barrier options and look-back options. In the context of this paper we concentrate on applications of occupation times of Brownian motion to problems in mathematical finance.

For instance the distribution of occupation times is decisive for the pricing of socalled $\alpha$-quantile options, a certain class of average options. Although occupation times of Brownian motion had been a subject of intensive research in stochastic calculus years ago (e.g. a nice proof of Lévy's famous arc-sine law is presented in Billingsley (1968)) the interest of financial economists and applied mathematicians in questions concerning quantile options has caused a renaissance in this topic [see Akahori (1995), Dassios (1995)].

This paper intends to present a survey and a summary of familiar and recent results with respect to the distribution of occupation times of Brownian motion (with drift) in Section 1. The main result of this section is Theorem 1.1, where a representation of the occupation times' density by the partial derivatives of a convolution integral is provided. In Section 2 we demonstrate how this represen- 
tation can be used to derive by elementary methods one of the most interesting results of recent research - Dassios' identity in law for occupation times. Then, in Section 3, a closed form representation of the distribution in question is introduced which is appropriate for the calculation of option pricing formulae in the Black \& Scholes framework. Such an application we present in Section 4 where the explicit formula of a quantile option is discussed. Finally, in the Appendix miscellaneous remarks and computational aspects of proofs are provided for those readers who are interested in technical details.

\section{OCCUPATION TIMES AND A GENERALIZED ARC-SINE LAW}

Let $\left(W_{t}, t \geq 0\right)$ be a one-dimensional standard Brownian motion. With $\nu \in g R$ we introduce the Brownian motion with drift $Z$ defined by $Z_{t}=W_{t}+\nu t, t \geq 0$. In the framework of this paper we consider the restriction of $Z$ to the time-interval $[0 ; T]$, i.e. $Z$ is a random variable with values in the measurable space $\left(C[0 ; T] ; \mathcal{C}^{[0 ; T]}\right)$, the space of all continuous functions on $[0 ; T]$ endowed with the $\sigma$-algebra of Borel sets [see Billingsley (1968), Chapter 2]. Then by application of the measurable functional $\Gamma_{+}(T, k): C[0 ; T] \rightarrow g R$ defined by

$$
\Gamma_{+}(T, k)(z):=\int_{t=0}^{T} \mathbf{1}[z(t)>k] d t, z \in C[0 ; T],
$$

to $Z$ we obtain the real-valued random variable occupation time $\Gamma_{+}(T, k)(Z)$ of $(k ; \infty)$ up to time $T$ [see Karatzas \& Shreve (1988), Example 3.6.2]. In the driftless case $\nu=0$ with $k=0$ the distribution of $\Gamma_{+}(T, 0)(W)$ is a familiar example in the literature on Brownian motion and provides a well-known arc-sine law. As an application of the central limit theorem of random walk Billingsley presents an elementary derivation for the joint density $P\left[\Gamma_{+}(T, 0)(W) \in d u ; W_{T} \in d x\right.$ ] [see Billingsley (1968), Chapter 2, pp. 80-83]. The joint density $P\left[\Gamma_{+}(T, 0)(W) \in d u ; W_{T} \in d x\right]$ is thus given by the formula,

$P\left[\Gamma_{+}(T, 0)(W) \in d u ; W_{T} \in d x\right]=\left\{\begin{array}{l}\frac{|x|}{2 \pi} \int_{t=u}^{T} \frac{\exp \left\{-\frac{x^{2}}{2(T-t)}\right\}}{[t(T-t)]^{\frac{3}{2}}} d t d u d x \quad, x<0 ; \\ \frac{|x|}{2 \pi} \int_{t=T-u}^{T} \frac{\exp \left\{-\frac{x^{2}}{2(T-t)}\right\}}{[t(T-t)]^{\frac{3}{2}}} d t d u d x, x>0 .\end{array}\right.$

With respect to the following explicit calculations of distributions and their applications in mathematical finance we denote the univariate and the bivariate standard normal distribution functions by

$$
\mathcal{N}(x)=\frac{1}{\sqrt{2 \pi}} \int_{v=-\infty}^{x} \exp \left\{-\frac{v^{2}}{2}\right\} d v
$$


for all $x \in g R$ and by

$\mathcal{N}(x, y ; \rho)=\frac{1}{2 \pi \sqrt{1-\rho^{2}}} \int_{v=-\infty}^{x} \int_{w=-\infty}^{y} \exp \left\{-\frac{1}{2\left(1-\rho^{2}\right)}\left(v^{2}-2 \rho v w+w^{2}\right)\right\} d w d v$

for all $x, y \in g R$ and all $\rho \in(-1 ; 1)$.

By Girsanov's theorem we obtain the joint density

$P\left[\Gamma_{+}(T, 0)(Z) \in d u ; Z_{T} \in d x\right]=\exp \left\{-\frac{\nu^{2}}{2} T+\nu x\right\} P\left[\Gamma_{+}(T, 0)(W) \in d u ; W_{T} \in d x\right]$

and by application of Fubini's theorem the first result of Akahori's generalized arc-sine law [see Akahori (1995), Theorem 1.1 (i)],

$$
\begin{aligned}
& P\left[\Gamma_{+}(T, 0)(Z) \in d u\right] \\
= & \left\{\frac{1}{\pi} \exp \left\{-\frac{\nu^{2}}{2} T\right\} \frac{1}{\sqrt{u(T-u)}}+\sqrt{\frac{2}{\pi}} \frac{\nu}{\sqrt{T-u}} \exp \left\{-\frac{\nu^{2}}{2}(T-u)\right\} \mathcal{N}(\nu \sqrt{u})\right. \\
& \left.-\sqrt{\frac{2}{\pi}} \frac{\nu}{\sqrt{u}} \exp \left\{-\frac{\nu^{2}}{2} u\right\} \mathcal{N}(-\nu \sqrt{T-u})-2 \nu^{2} \mathcal{N}(\nu \sqrt{u}) \mathcal{N}(-\nu \sqrt{T-u})\right\} d u
\end{aligned}
$$

Actually for $\nu=0$ this result yields Lévy's classical arc-sine law for occupation times

$$
P\left[\Gamma_{+}(T, 0)(W) \leq u\right]=\frac{1}{\pi} \int_{t=0}^{u} \frac{d t}{\sqrt{t(T-t)}}=\frac{2}{\pi} \arcsin \sqrt{\frac{u}{T}}
$$

for $0 \leq u \leq T$.

With eq. (3) we immediately obtain a double integral representation for $P\left[\Gamma_{+}(T, k)(Z) \leq t\right]$. First we introduce the functional passage time $\mathrm{T}_{k}$ by

$$
\mathrm{T}_{k}(z)=\inf \left\{t \geq 0 \mid z_{t}=k\right\}
$$

and consider $\mathrm{T}_{k}(Z)$ with distribution

$$
\begin{aligned}
P\left[\mathrm{~T}_{k}(Z) \leq t\right]=P\left[\min _{0 \leq s \leq t} Z_{s} \leq k\right]= & \mathcal{N}\left(\frac{k}{\sqrt{t}}-\nu \sqrt{t}\right)+ \\
& \exp \{2 k \nu\} \mathcal{N}\left(\frac{k}{\sqrt{t}}+\nu \sqrt{t}\right)
\end{aligned}
$$

for $k<0$ and with distribution

$$
\begin{aligned}
P\left[\mathrm{~T}_{k}(Z) \leq t\right]=P\left[\max _{0 \leq s \leq t} Z_{s} \geq k\right]= & \mathcal{N}\left(-\frac{k}{\sqrt{t}}+\nu \sqrt{t}\right)+ \\
& \exp \{2 k \nu\} \mathcal{N}\left(-\frac{k}{\sqrt{t}}-\nu \sqrt{t}\right)
\end{aligned}
$$


for $k>0$. Obviously the following density formula is valid.

$$
P\left[\mathrm{~T}_{k}(Z) \in d t\right]=h(t, k ; \nu) d t,
$$

where $h(t, k ; \nu)=\frac{|k|}{\sqrt{2 \pi t^{3}}} \exp \left\{-\frac{(k-\nu t)^{2}}{2 t}\right\}$ [see Karatzas \& Shreve (1988), p. $196 f]$. Furthermore we denote

$$
\phi(T, t ; \nu)=P\left[\Gamma_{+}(T, 0)(Z) \leq t\right], 0 \leq t \leq T,
$$

and since $Z$ has independent increments we have for all $k<0$ and all $0 \leq t<T$ the relation

$$
P\left[\Gamma_{+}(T, k)(Z) \leq t\right]=\int_{s=0}^{t} \phi(T-s, t-s ; \nu) h(s, k ; \nu) d s,
$$

and by the obvious identity in law

$$
\Gamma_{+}(T, k)(W(\cdot)+\nu \cdot) \stackrel{\mathcal{D}}{=} T-\Gamma_{+}(T,-k)(W(\cdot)-\nu \cdot)
$$

the corresponding relation for all $k>0$ and all $0 \leq t \leq T$

$$
P\left[\Gamma_{+}(T, k)(Z) \leq t\right]=1-\int_{s=0}^{T-t} \phi(T-s, T-t-s ;-\nu) h(s,-k ;-\nu) d s .(7)
$$

Eq.s (6) and (7) correspond with Akahori's Theorem 1.1 (ii) [see Akahori (1995)].

As our first original result in this paper we now present a single integral version for the distribution of $\Gamma_{+}(T, k)(Z)$ and thus an explicit formula for its density. For values $0 \leq \theta \leq \tau, \kappa, \mu \in g R$ we define the functions $F(\tau, \theta, \kappa ; \mu)$ and $D(\tau, \theta, \kappa ; \mu)$ by

$$
F(\tau, \theta, \kappa ; \mu)=\int_{s=0}^{\theta} \phi(\tau-s, \theta-s ; \mu) h(s, \kappa ; \mu) d s
$$

and by

$$
D(\tau, \theta, \kappa ; \mu)=\int_{s=0}^{\theta} \mathcal{N}(-\mu \sqrt{\tau-s}) \mathcal{N}\left(-\operatorname{sign}(\kappa)\left(\frac{\kappa}{\sqrt{s}}+\mu \sqrt{s}\right)\right) d s .
$$

We shall demonstrate in the following theorem that the distribution of occupation times of Brownian motion with drift can be described completely by the integral $D$ and its partial derivatives with respect to $\kappa$ and $\tau$.

Theorem 1.1. Let $F$ and $D$ be defined as in eqs. (8) and (9). Then the distribution of $\Gamma_{+}(T, k)(Z)$ is determined on $[0 ; T)$ by

$$
\begin{array}{rr}
P\left[\Gamma_{+}(T, k)(Z) \leq t\right]=r(T, t, k ; \nu), & k<0 \\
P\left[\Gamma_{+}(T, k)(Z) \leq t\right]=1-F(T, T-t,-k ;-\nu), & k>0,
\end{array}
$$


and for $\kappa<0$ the function $F$ can be represented in the following way.

$$
\begin{aligned}
F(\tau, \theta, \kappa ; \mu)= & -2 \mu^{2} \exp \{2 \kappa \mu\} D(\tau, \theta, \kappa ; \mu)-2 \mu \exp \{2 \kappa \mu\} \frac{\partial}{\partial \kappa} D(\tau, \theta, \kappa ; \mu) \\
& -4 \exp \{2 \kappa \mu\} \frac{\partial}{\partial \tau} D(\tau, \theta, \kappa ; \mu)-\frac{4}{\mu} \exp \{2 \kappa \mu\} \frac{\partial^{2}}{\partial \kappa \partial \tau} D(\tau, \theta, \kappa ; \mu) .
\end{aligned}
$$

For $t<0$ we trivially have $P\left[\Gamma_{+}(T, k)(Z) \leq t\right]=0$ and for $t \geq T$ we have $P\left[\Gamma_{+}(T, k)(Z) \leq t\right]=1$.

Proof. For $\kappa<0$ we rewrite eq. (8) as

$$
F(\tau, \theta, \kappa ; \mu)=\int_{s=0}^{\theta} \int_{u=0}^{\theta-s} \frac{\partial}{\partial u} \phi(\tau-s, u ; \mu) h(s, \kappa ; \mu) d u d s
$$

and obtain the partial derivative of $F$ with respect to $\theta$ by

$$
\frac{\partial}{\partial \theta} F(\tau, \theta, \kappa ; \mu)=\int_{s=0}^{\theta} \frac{\partial}{\partial u} \phi(\tau-s, \theta-s ; \mu) h(s, \kappa ; \mu) d s
$$

where $\frac{\partial}{\partial u} \phi(\tau-s, u ; \mu)$ is determined by eq. (3). Thus we have the following representation for $\frac{\partial}{\partial \theta} F(\tau, \theta, \kappa ; \mu)$.

$$
\frac{\partial}{\partial \theta} F(\tau, \theta, \kappa ; \mu)=\sum_{i=1}^{4} G_{i}(\tau, \theta, \kappa ; \mu)
$$

where we define

$$
\begin{aligned}
& J(\theta, \kappa ; \mu) \quad=\int_{s=0}^{\theta} \frac{\kappa}{\sqrt{2 \pi s^{3}}} \mathcal{N}(\mu \sqrt{\theta-s}) \exp \left\{-\frac{(\kappa-\mu s)^{2}}{2 s}\right\} d s, \\
& G_{1}(\tau, \theta, \kappa ; \mu)=2 \mu^{2} \mathcal{N}(-\mu \sqrt{\tau-\theta}) J(\theta, \kappa ; \mu), \\
& G_{2}(\tau, \theta, \kappa ; \mu)=4 \mathcal{N}(-\mu \sqrt{\tau-\theta})\left\{\frac{\partial}{\partial \theta} J(\theta, \kappa ; \mu)-\frac{\kappa}{2 \sqrt{2 \pi \theta^{3}}} \exp \left\{-\frac{(\kappa-\mu \theta)^{2}}{2 \theta}\right\}\right\}, \\
& G_{3}(\tau, \theta, \kappa ; \mu)=4 \frac{\partial}{\partial \tau} \mathcal{N}(-\mu \sqrt{\tau-\theta}) J(\theta, \kappa ; \mu), \\
& G_{4}(\tau, \theta, \kappa ; \mu)=\frac{8}{\mu^{2}} \frac{\partial}{\partial \tau} \mathcal{N}(-\mu \sqrt{\tau-\theta})\left\{\frac{\partial}{\partial \theta} J(\theta, \kappa ; \mu)-\frac{\kappa}{2 \sqrt{2 \pi \theta^{3}}} \exp \left\{-\frac{(\kappa-\mu \theta)^{2}}{2 \theta}\right\}\right\} .
\end{aligned}
$$

We only have to calculate $J(\theta, \kappa ; \mu)$ and by partial differentiation we obtain explicit versions for $G_{i}(\tau, \theta, \kappa ; \mu), 1 \leq i \leq 4$, and thus for $\frac{\partial}{\partial \theta} F(\tau, \theta, \kappa ; \mu)$.

$$
\text { Since } \frac{\partial}{\partial \theta} J(\theta, \kappa ; \mu)=\frac{1}{2 \sqrt{2 \pi}} \exp \{2 \kappa \mu\}\left(\frac{\kappa}{\theta^{\frac{3}{2}}}-\frac{\mu}{\theta^{\frac{1}{2}}}\right) \exp \left\{-\frac{1}{2}\left(\frac{\kappa}{\sqrt{\theta}}+\mu \sqrt{\theta}\right)^{2}\right\} \text { we }
$$
have

$$
J(\theta, \kappa ; \mu)=-\exp \{2 \kappa \mu\} \mathcal{N}\left(\frac{\kappa}{\sqrt{\theta}}+\mu \sqrt{\theta}\right)
$$


We set

$$
d(\tau, \theta, \kappa ; \mu):=\frac{\partial}{\partial \theta} D(\tau, \theta, \kappa ; \mu)=\mathcal{N}(-\mu \sqrt{\tau-\theta}) \mathcal{N}\left(\frac{\kappa}{\sqrt{\theta}}+\mu \sqrt{\theta}\right),
$$

and obtain the assertion of Theorem 1.1 immediately.

REMARK 1.2. The joint density in eq. (2) can be expressed explicitly by

$$
\begin{aligned}
& P\left[\Gamma_{+}(T, 0)(W) \in d u, W_{T} \in d x\right] \\
= & \left\{-\frac{x}{\pi T^{2}} \sqrt{\frac{T-u}{u}} \exp \left\{-\frac{x^{2}}{2(T-u)}\right\}+\right. \\
& \left.\sqrt{\frac{2}{\pi}} \exp \left\{-\frac{x^{2}}{2 T}\right\} \frac{T-x^{2}}{T^{\frac{5}{2}}} \mathcal{N}\left(\frac{x}{\sqrt{T}} \sqrt{\frac{u}{T-u}}\right)\right\} d u d x
\end{aligned}
$$

for $x<0$ and by

$$
\begin{aligned}
& P\left[\Gamma_{+}(T, 0)(W) \in d u, W_{T} \in d x\right] \\
= & \left\{\frac{x}{\pi T^{2}} \sqrt{\frac{u}{T-u}} \exp \left\{-\frac{x^{2}}{2 u}\right\}+\right. \\
& \left.\sqrt{\frac{2}{\pi}} \exp \left\{-\frac{x^{2}}{2 T}\right\} \frac{T-x^{2}}{T^{\frac{5}{2}}} \mathcal{N}\left(-\frac{x}{\sqrt{T}} \sqrt{\frac{T-u}{u}}\right)\right\} d u d x
\end{aligned}
$$

for $x>0$.

An alternative approach to the distribution of occupation times was presented by Karatzas \& Shreve $(1984,1988)$ who provided a density formula for

$$
P\left[\Gamma_{+}(T, 0)(W) \in d u, W_{T} \in d x, \mathrm{~L}_{T}(W) \in d b\right]
$$

where L denotes the local time of Brownian motion [see Karatzas \& Shreve (1988), Proposition 6.3.9].

REMARK 1.3. For convenience of some calculations the partial derivatives of $d(\tau, \theta, \kappa ; \mu)$ are provided.

$$
\begin{aligned}
\frac{\partial}{\partial \kappa} d(\tau, \theta, \kappa ; \mu) & =\frac{1}{\sqrt{2 \pi \theta}} \exp \left\{-\frac{1}{2}\left(\frac{\kappa}{\sqrt{\theta}}+\mu \sqrt{\theta}\right)^{2}\right\} \mathcal{N}(-\mu \sqrt{\tau-\theta}), \\
\frac{\partial}{\partial \tau} d(\tau, \theta, \kappa ; \mu) & =-\frac{\mu}{2 \sqrt{2 \pi(\tau-\theta)}} \exp \left\{-\frac{\mu^{2}}{2}(\tau-\theta)\right\} \mathcal{N}\left(\frac{\kappa}{\sqrt{\theta}}+\mu \sqrt{\theta}\right), \\
\frac{\partial^{2}}{\partial \kappa \partial \tau} d(\tau, \theta, \kappa ; \mu) & =-\frac{\mu}{4 \pi \sqrt{\theta(\tau-\theta)}} \exp \left\{-\frac{\kappa^{2}}{2 \theta}-\kappa \mu-\frac{\mu^{2}}{2} \tau\right\} .
\end{aligned}
$$

REMARK 1.4. Though in Akahori (1995) a different notation is used it is evident that in Akahori's Theorem 1.1 (ii) are some slight incorrectnesses beside a few minor 
typographic errors. Otherwise $F(T, t, k ; \nu)=1-F(T, T-t,-k ;-\nu)$ would hold for all values $k>0, \nu \in g R$ and all $0 \leq t \leq T$. To demonstrate this contradiction choose $\nu=0$ and prove by using Remark A.2 that in general

$$
\frac{\partial}{\partial \theta} F(T, t, k ; 0) \neq \frac{\partial}{\partial \theta} F(T, T-t,-k ; 0) .
$$

REMARK 1.5. The function $P\left[\Gamma_{+}(T, k)(Z) \leq t\right]$ is continuous in the crucial point $k=0$, thus it is continuous for all $k \in g R$. This can be easily proved by Theorem 1.1, Remark 1.3 and eq. (3).

Furthermore $P\left[\Gamma_{+}(T, k)(Z) \leq t\right]$ is continuous for all $t \neq T$ if $k<0$ since

$$
P\left[\Gamma_{+}(T, k)(Z)=T\right]=P\left[\mathrm{~T}_{k}(Z) \geq T\right]>0 .
$$

Analogously $P\left[\Gamma_{+}(T, k)(Z) \leq t\right]$ is continuous for all $t \neq 0$ if $k>0$. Evidently it is continuous on $g R$ for $k=0$.

\section{A STRAIGHTFORWARD PROOF FOR DASSIOS' IDENTITY IN LAW}

A simple but remarkable relationship between the distribution of occupation times and passage times was discovered by Dassios [see Dassios (1995)] who pursued the investigations of Akahori on the so-called $\alpha$-quantile or $\alpha$-percentile options, a problem in mathematical finance which we shall illuminate in Section 4 . In the language of this paper we present the result of Dassios in the following way.

$$
\begin{aligned}
& P\left[\int_{s=0}^{T} \mathbf{1}\left[W_{s}+\nu s>k\right] d s \leq t\right] \\
= & P\left[\max _{0 \leq s \leq T-t}\left\{W_{s}+\nu s\right\}+\min _{0 \leq s \leq t}\left\{W_{s}^{*}+\nu s\right\} \leq k\right]
\end{aligned}
$$

for all $k \in g R$ and all $t \in[0 ; T]$ where $W$ and $W^{*}$ are independent standard Brownian motions. Dassios proved this property using Feynman \& Kac computations, two further proofs based on stochastic properties of Brownian motion were provided by Embrechts, Rogers and Yor (1995).

In this paper we point out a direct proof of Dassios' identity in law using the explicit density of $\Gamma_{+}(T, k)(Z)$ and the knowledge of the distribution of passage times. Before we prove eq. (10) we provide a representation of $\frac{\partial}{\partial \theta} F(\tau, \theta, \kappa ; \mu)$ as a convolution integral. Referring to this convolution integral Dassios' result can be derived by relatively elementary methods.

Proof of Dassios' theorem. By Theorem 1.1 the following representation holds for $\kappa<0$, 


$$
\frac{\partial}{\partial \theta} F(\tau, \theta, \kappa ; \mu)=g_{1}(\tau, \theta, \kappa ; \mu)+g_{2}(\tau, \theta, \kappa ; \mu)
$$

where

$$
\begin{aligned}
g_{1}(\tau, \theta, \kappa ; \mu)= & -2 \exp \{2 \kappa \mu\}\left\{\mu^{2} d(\tau, \theta, \kappa ; \mu)+\mu \frac{\partial}{\partial \kappa} d(\tau, \theta, \kappa ; \mu)+\right. \\
& \left.2 \frac{\partial}{\partial \tau} d(\tau, \theta, \kappa ; \mu)\right\}, \\
g_{2}(\tau, \theta, \kappa ; \mu)= & -\frac{4}{\mu} \exp \{2 \kappa \mu\} \frac{\partial^{2}}{\partial \kappa \partial \tau} d(\tau, \theta, \kappa ; \mu) .
\end{aligned}
$$

Since $d(\tau, \theta, \kappa ; \mu)$ is a product of two functions converging to 0 for $\kappa \rightarrow-\infty$ it can be interpreted as a convolution integral.

$$
\begin{aligned}
& d(\tau, \theta, \kappa ; \mu)=\mathcal{N}(-\mu \sqrt{\tau-\theta}) \mathcal{N}\left(\frac{\kappa}{\sqrt{\theta}}+\mu \sqrt{\theta}\right) \\
= & \int_{z=-\infty}^{\kappa} \frac{\partial}{\partial z} \mathcal{N}\left(\frac{z}{\sqrt{\theta}}+\mu \sqrt{\theta}\right) \mathcal{N}\left(-\frac{\kappa-z}{\sqrt{\tau-\theta}}-\mu \sqrt{\tau-\theta}\right) d z \\
& +\int_{z=-\infty}^{\kappa} \frac{\partial}{\partial z} \mathcal{N}\left(-\frac{\kappa-z}{\sqrt{\tau-\theta}}-\mu \sqrt{\tau-\theta}\right) \mathcal{N}\left(\frac{z}{\sqrt{\theta}}+\mu \sqrt{\theta}\right) d z .
\end{aligned}
$$

Partial differentiation with respect to $\kappa$ yields the representation of $\frac{\partial}{\partial \kappa} d(\tau, \theta, \kappa ; \mu)$ as convolution. The following relation is the crucial idea of this proof. It is obtained by integration by parts and Remark 1.3 to $\frac{\partial}{\partial \tau} d(\tau, \theta, \kappa ; \mu)$, namely

$$
\begin{aligned}
\mu \frac{\partial}{\partial \kappa} d(\tau, \theta, \kappa ; \mu)= & \mu \int_{z=-\infty}^{\kappa} \frac{\partial^{2}}{(\partial z)^{2}} \mathcal{N}\left(\frac{z}{\sqrt{\theta}}+\mu \sqrt{\theta}\right) \mathcal{N}\left(-\frac{\kappa-z}{\sqrt{\tau-\theta}}-\mu \sqrt{\tau-\theta}\right) d z \\
& -\mu \int_{z=-\infty}^{\kappa} \frac{\partial^{2}}{(\partial z)^{2}} \mathcal{N}\left(-\frac{\kappa-z}{\sqrt{\tau-\theta}}-\mu \sqrt{\tau-\theta}\right) \mathcal{N}\left(\frac{z}{\sqrt{\theta}}+\mu \sqrt{\theta}\right) d z \\
& -2 \frac{\partial}{\partial \tau} d(\tau, \theta, \kappa ; \mu) .
\end{aligned}
$$

We arrive at

$$
\begin{aligned}
& g_{1}(\tau, \theta, \kappa ; \mu) \\
= & 2 \mu \int_{z=-\infty}^{\kappa} \frac{\kappa-z}{\sqrt{2 \pi(\tau-\theta)^{3}}} \exp \left\{-\frac{1}{2}\left(\frac{\kappa-z}{\sqrt{\tau-\theta}}-\mu \sqrt{\tau-\theta}\right)^{2}\right\} . \\
& \exp \{2 z \mu\} \mathcal{N}\left(\frac{z}{\sqrt{\theta}}+\mu \sqrt{\theta}\right) d z+2 \mu \int_{z=-\infty}^{\kappa} \frac{z}{\sqrt{2 \pi \theta^{3}}} \exp \left\{-\frac{1}{2}\left(\frac{z}{\sqrt{\theta}}-\mu \sqrt{\theta}\right)^{2}\right\} .
\end{aligned}
$$




$$
\exp \{2(\kappa-z) \mu\} \mathcal{N}\left(-\frac{\kappa-z}{\sqrt{\tau-\theta}}-\mu \sqrt{\tau-\theta}\right) d z
$$

Similarly $\frac{\partial^{2}}{\partial \tau \partial \kappa} d(\tau, \theta, \kappa ; \mu)=\frac{\partial}{\partial \tau} \mathcal{N}(-\mu \sqrt{\tau-\theta}) \frac{\partial}{\partial \kappa} \mathcal{N}\left(\frac{\kappa}{\sqrt{\theta}}+\mu \sqrt{\theta}\right)$ can be represented as convolution. We obtain

$$
\begin{aligned}
& g_{2}(\tau, \theta, \kappa ; \mu) \\
= & \int_{z=-\infty}^{\kappa} \frac{\kappa-z}{\sqrt{2 \pi(\tau-\theta)^{3}}} \exp \left\{-\frac{1}{2}\left(\frac{\kappa-z}{\sqrt{\tau-\theta}}-\mu \sqrt{\tau-\theta}\right)^{2}\right\} . \\
& \frac{2}{\sqrt{2 \pi \theta}} \exp \left\{-\frac{1}{2}\left(\frac{z}{\sqrt{\theta}}-\mu \sqrt{\theta}\right)^{2}\right\} d z-\int_{z=-\infty}^{\kappa} \frac{z}{\sqrt{2 \pi \theta^{3}}} \exp \left\{-\frac{1}{2}\left(\frac{z}{\sqrt{\theta}}-\mu \sqrt{\theta}\right)^{2}\right\} . \\
& \frac{2}{\sqrt{2 \pi(\tau-\theta)}} \exp \left\{-\frac{1}{2}\left(\frac{\kappa-z}{\sqrt{\tau-\theta}}-\mu \sqrt{\tau-\theta}\right)^{2}\right\} d z .
\end{aligned}
$$

Since by eqs. (4) and (5)

$$
\begin{aligned}
P\left[\min _{0 \leq s \leq \theta}\left\{W_{s}^{*}+\mu s\right\} \in d z\right]= & 2 \mu \exp \{2 z \mu\} \mathcal{N}\left(\frac{z}{\sqrt{\theta}}+\mu \sqrt{\theta}\right) d z \\
& +\frac{2}{\sqrt{2 \pi \theta}} \exp \left\{-\frac{1}{2}\left(\frac{z}{\sqrt{\theta}}-\mu \sqrt{\theta}\right)^{2}\right\} d z, \\
P\left[\max _{0 \leq s \leq \tau-\theta}\left\{W_{s}+\mu s\right\} \in d(\kappa-z)\right]= & 2 \mu \exp \{2(\kappa-z) \mu\} \mathcal{N}\left(-\frac{\kappa-z}{\sqrt{\tau-\theta}}-\mu \sqrt{\tau-\theta}\right) d z \\
& -\frac{2}{\sqrt{2 \pi(\tau-\theta)}} \exp \left\{-\frac{1}{2}\left(\frac{\kappa-z}{\sqrt{\tau-\theta}}-\mu \sqrt{\tau-\theta}\right)^{2}\right\} d z, \\
\frac{\partial}{\partial \theta} P\left[\min _{0 \leq s \leq \theta}\left\{W_{s}^{*}+\mu s\right\} \leq z\right]= & -\frac{z}{\sqrt{2 \pi \theta^{3}}} \exp \left\{-\frac{1}{2}\left(\frac{z}{\sqrt{\theta}}-\mu \sqrt{\theta}\right)^{2}\right\}, \\
\frac{\partial}{\partial \theta} P\left[\max _{0 \leq s \leq \tau-\theta}\left\{W_{s}+\mu s\right\} \leq \kappa-z\right]= & \frac{\kappa-z}{\sqrt{2 \pi(\tau-\theta)^{3}}} \exp \left\{-\frac{1}{2}\left(\frac{\kappa-z}{\sqrt{\tau-\theta}}-\mu \sqrt{\tau-\theta}\right)^{2}\right\},
\end{aligned}
$$

we obtain a final convolution integral representation by

$$
\begin{aligned}
& \frac{\partial}{\partial \theta} F(\tau, \theta, \kappa ; \mu)=\frac{\partial}{\partial \theta} P\left[\int_{s=0}^{\tau} \mathbf{1}\left[W_{s}+\mu s>\kappa\right] d s \leq \theta\right] \\
= & \int_{z=-\infty}^{\kappa} \frac{\partial}{\partial \theta} P\left[\max _{0 \leq s \leq \tau-\theta}\left\{W_{s}+\mu s\right\} \leq \kappa-z\right] P\left[\min _{0 \leq s \leq \theta}\left\{W_{s}^{*}+\mu s\right\} \in d z\right] \\
& -\int_{z=-\infty}^{\kappa} \frac{\partial}{\partial \theta} P\left[\min _{0 \leq s \leq \theta}\left\{W_{s}^{*}+\mu s\right\} \leq z\right] P\left[\max _{0 \leq s \leq \tau-\theta}\left\{W_{s}+\mu s\right\} \in d(\kappa-z)\right] \\
= & \frac{\partial}{\partial \theta} P\left[\max _{0 \leq s \leq \tau-\theta}\left\{W_{s}+\mu s\right\}+\min _{0 \leq s \leq \theta}\left\{W_{s}^{*}+\mu s\right\} \leq \kappa\right] .
\end{aligned}
$$


From this and by the fact that eq. (10) holds trivially for $k<0$ and $t=0$ eq. (10) is generally valid for $k<0$. For $k \geq 0$ we use Theorem 1.1 and obtain

$$
\begin{array}{r}
P\left[\int_{s=0}^{T} \mathbf{1}\left[W_{s}+\nu s>k\right] d s \leq t\right] \\
=1-P\left[\int_{s=0}^{T} \mathbf{1}\left[-W_{s}-\nu s>-k\right] d s \leq T-t\right] \\
=1-P\left[\max _{0 \leq s \leq t}\left\{-W_{s}^{*}-\nu s\right\}+\min _{0 \leq s \leq T-t}\left\{-W_{s}-\nu s\right\} \leq-k\right] \\
=P\left[\max _{0 \leq s \leq T-t}\left\{W_{s}+\nu s\right\}+\min _{0 \leq s \leq t}\left\{W_{s}^{*}+\nu s\right\} \leq k\right] .
\end{array}
$$

This completes the proof.

\section{A CLOSED FORM DISTRIBUTION FUNCTION}

In Section 1, Theorem 1.1, we provided a single integral version for the distribution of $\Gamma_{+}(T, k)(Z)$ and thus an explicit representation for its density. With respect to various applications in mathematical finance an explicit formula for this distribution would be a comfortable result. By eq. (8) and Theorem 1.1 it is sufficient to obtain an explicit version of the function $F$.

Theorem 3.1. The function $F$ defined by eq. (8) can be represented explicitly for all values $\kappa<0$ in the following way.

$$
\begin{aligned}
F(\tau, \theta, \kappa ; \mu)= & \left\{3+2 \kappa \mu+2 \mu^{2} \tau\right\} \exp \{2 \kappa \mu\} \mathcal{N}\left(\frac{\kappa}{\sqrt{\tau}}+\mu \sqrt{\tau},-\mu \sqrt{\tau-\theta}\right. \\
& \left.-\sqrt{1-\frac{\theta}{\tau}}\right) \\
& +\mathcal{N}\left(\frac{\kappa}{\sqrt{\tau}}-\mu \sqrt{\tau}, \mu \sqrt{\tau-\theta} ;-\sqrt{1-\frac{\theta}{\tau}}\right) \\
& -\left\{1+2 \kappa \mu+2 \mu^{2} \theta\right\} \exp \{2 \kappa \mu\} \mathcal{N}\left(\frac{\kappa}{\sqrt{\theta}}+\mu \sqrt{\theta}\right) \mathcal{N}(-\mu \sqrt{\tau-\theta}) \\
& +\mathcal{N}\left(\frac{\kappa}{\sqrt{\theta}}-\mu \sqrt{\theta}\right) \mathcal{N}(-\mu \sqrt{\tau-\theta}) \\
& -2 \mu \sqrt{\frac{\theta}{2 \pi}} \exp \left\{-\frac{1}{2}\left(\frac{\kappa}{\sqrt{\theta}}-\mu \sqrt{\theta}\right)^{2}\right\} \mathcal{N}(-\mu \sqrt{\tau-\theta}) \\
& +2 \mu \sqrt{\frac{\tau}{2 \pi}} \exp \left\{-\frac{1}{2}\left(\frac{\kappa}{\sqrt{\tau}}-\mu \sqrt{\tau}\right)^{2}\right\} \mathcal{N}\left(\kappa \sqrt{\frac{1}{\theta}-\frac{1}{\tau}}\right)
\end{aligned}
$$




$$
-2 \mu \sqrt{\frac{\tau-\theta}{2 \pi}} \exp \left\{-\frac{1}{2} \mu^{2}(\tau-\theta)\right\} \exp \{2 \kappa \mu\} \mathcal{N}\left(\frac{\kappa}{\sqrt{\theta}}+\mu \sqrt{\theta}\right) .
$$

Since the proof of Theorem 3.1 is very technical with respect to the explicit calculation of $F(\tau, \theta, \kappa ; \mu)$ we omit it here and refer the reader interested in the details of the proof to the appendix of the paper.

\section{THE PRICE OF A QUANTILE OPTION}

In the last few years a lot of problems concerning the application of measurable functionals to Brownian motion with drift have been stimulated by the rapid development of mathematical finance based on the fundamental ideas of Black \& Scholes. In this paper we do not want to discuss economic models and the reader interested in those questions should be referred to a number of excellent surveys on option pricing theory (e.g. Harrison \& Pliska (1981), Duffie (1988), et al.).

For our purposes it is sufficient to know that an option is determined uniquely by a measurable functional $\Phi$ on a certain underlying security $S$ with initial price $S_{0}$, the so-called payout profile, and that the price of this option can be calculated in the Black \& Scholes model by the discounted expectation

$$
r^{-T} E \Phi(X)
$$

using the no-arbitrage price-process $X$ which is described by

$$
X_{t}=S_{0} \exp \left\{\sigma W_{t}+\mu_{B S} t\right\}, 0 \leq t \leq T,
$$

where $[0 ; T]$ is the considered time-horizon, $\sigma$ the volatility of the underlying security, $r(>1)$ is one plus the risk-free interest rate and

$$
\mu_{B S}=\log r-\frac{1}{2} \sigma^{2}
$$

the risk-neutral drift determined by the assumptions of Black \& Scholes.

The option types we want to discuss here are so-called average options, especially the class of $\alpha$-quantile options or - in Akahori's terminology - $\alpha$-percentile options. As mentioned before Dassios found his identity in law when investigating $\alpha$-quantile options. To illustrate the properties of such an option let $z$ be a continuous function on the time-interval $\left[-T_{0} ; T\right]$ with $T_{0}, T \geq 0$. Then for $0<\alpha<1$ the $\alpha$-quantile $M\left(\alpha, T_{0}+T\right)$ of $z$ is defined by

$$
M\left(\alpha, T_{0}+T\right)(z)=\inf \left\{k \in g R \mid \int_{\tau=-T_{0}}^{T} \mathbf{1}[z(\tau) \leq k] d \tau>\alpha\left(T_{0}+T\right)\right\} .
$$

Now we represent the price-process $S$ of the underlying security by

$$
S_{\tau}=S_{0} \exp \{\sigma s(\tau)\},-T_{0} \leq \tau \leq T,
$$


where the values $S_{\tau}$ respectively $s(\tau)$ in $\left[-T_{0} ; 0\right]$ are given by historical data. Given a certain strike $K$ the payout profile $\Phi_{0}$ of an $\alpha$-quantile (call) option is described by

$$
\begin{aligned}
\Phi_{0}(S) & =\left(M\left(\alpha, T_{0}+T\right)(S)-K\right)^{+} \\
& =\left(S_{0} \exp \left\{\sigma M\left(\alpha, T_{0}+T\right)(s)\right\}-K\right)^{+}
\end{aligned}
$$

using the identity

$$
M\left(\alpha, T_{0}+T\right)(S)=S_{0} \exp \left\{\sigma M\left(\alpha, T_{0}+T\right)(s)\right\} .
$$

For pricing an option dependent on the future time-interval $[0 ; T]$ in the Black \& Scholes model we use the process $X$ and define $X_{\tau}=S_{\tau}$ for all $\tau \in\left[-T_{0} ; 0\right]$. Then the price of this $\alpha$-quantile option is computed by

$$
\pi_{B S}\left(\Phi_{0}\right)=r^{-T} E\left(S_{0} \exp \left\{\sigma M\left(\alpha, T_{0}+T\right)(Z)\right\}-K\right)^{+}
$$

with $Z$ defined by $s$ on $\left[-T_{0} ; 0\right]$ and by $Z_{\tau}=W_{\tau}+\frac{\mu_{B S}}{\sigma} \tau$ for all $\tau \in[0 ; T]$. By the evident relation

$$
\left[M\left(\alpha, T_{0}+T\right)(Z) \leq k\right]=\left[\int_{\tau=-T_{0}}^{T} \mathbf{1}\left[Z_{\tau}>k\right] d \tau \leq(1-\alpha)\left(T_{0}+T\right)\right]
$$

we obtain the connection between $\alpha$-quantiles and occupation times. Furthermore the function $\gamma_{+}\left(T_{0}, k\right):=\int_{\tau=-T_{0}}^{0} \mathbf{1}\left[Z_{\tau}>k\right] d \tau$ is a deterministic function in $k$. Since $Z$ is considered to be a continuous function $\gamma_{+}\left(T_{0}, k\right)$ is strictly decreasing on the closed interval range $(Z)=\left\{Z_{\tau} \mid-T_{0} \leq \tau \leq 0\right\}$. For all values $k<\min _{-T_{0} \leq \tau \leq 0}\left\{Z_{\tau}\right\}$ we have $\gamma_{+}\left(T_{0}, k\right)=T_{0}$ and for all values $k \geq \max _{-T_{0} \leq \tau \leq 0}\left\{Z_{\tau}\right\}$ we have $\gamma_{+}\left(\bar{T}_{0}, k\right)=0$. Finally $\gamma_{+}\left(T_{0}, k\right)$ is right-continuous in $k$ with left limits.

Defining $t\left(T_{0}, k\right)$ by

$$
t\left(T_{0}, k\right)=(1-\alpha)\left(T_{0}+T\right)-\gamma_{+}\left(T_{0}, k\right)
$$

we have the property

$$
\left[M\left(\alpha, T_{0}+T\right)(Z) \leq k\right]=\left[\Gamma_{+}(T, k)(Z) \leq t\left(T_{0}, k\right)\right] .
$$

Then we obtain the distribution of $M\left(\alpha, T_{0}+T\right)(Z)$,

$$
F_{\alpha}(k):=P\left[M\left(\alpha, T_{0}+T\right)(Z) \leq k\right]=P\left[\Gamma_{+}(T, k)(Z) \leq t\left(T_{0}, k\right)\right],
$$

and by integration by parts we arrive at the integral version of the option's price,

$$
\pi_{B S}\left(\Phi_{0}\right)=r^{-T} S_{0} \sigma \int_{k>\frac{1}{\sigma} \log \frac{K}{S_{0}}}\left(1-F_{\alpha}(k)\right) \exp \{\sigma k\} d k .
$$


Since $F_{\alpha}$ depends on the arbitrary character of the historical function $t\left(T_{0}, k\right)$ the further computation of the $\alpha$-quantile option's price will be in general a question of numerical calculus. Nevertheless some properties of the option's price shall be discussed. To do so we introduce the following boundaries for $F_{\alpha}$, namely $\underline{k}\left(T_{0}\right)=\sup \left\{k \in g R \mid t\left(T_{0}, k\right)<0\right\}$ and $\bar{k}\left(T_{0}\right)=\inf \left\{k \in g R \mid t\left(T_{0}, k\right)>T\right\}$, such that $F_{\alpha}$ is concentrated on $\left[\underline{k}\left(T_{0}\right) ; \bar{k}\left(T_{0}\right)\right]$.

Then we have for $\frac{1}{\sigma} \log \frac{K}{S_{0}} \leq \underline{k}\left(T_{0}\right) \leq \bar{k}\left(T_{0}\right)$

$$
\begin{aligned}
\pi_{B S}\left(\Phi_{0}\right)= & r^{-T} S_{0} \sigma \int_{k=\frac{1}{\sigma} \log \frac{K}{S_{0}}}^{\bar{k}\left(T_{0}\right)}\left(1-F_{\alpha}(k)\right) \exp \{\sigma k\} d k \\
= & r^{-T}\left(S_{0} \exp \left\{\sigma \underline{k}\left(T_{0}\right)\right\}-K\right)+ \\
& r^{-T} S_{0} \sigma \int_{k \in\left[\underline{k}\left(T_{0}\right) ; \bar{k}\left(T_{0}\right)\right]}\left(1-F_{\alpha}(k)\right) \exp \{\sigma k\} d k,
\end{aligned}
$$

respectively for $\underline{k}\left(T_{0}\right)<\frac{1}{\sigma} \log \frac{K}{S_{0}}<\bar{k}\left(T_{0}\right)$

$$
\pi_{B S}\left(\Phi_{0}\right)=r^{-T} S_{0} \sigma \int_{k \in\left(\frac{1}{\sigma} \log \frac{K}{S_{0}} ; \bar{k}\left(T_{0}\right)\right]}\left(1-F_{\alpha}(k)\right) \exp \{\sigma k\} d k
$$

It is evident that for $\bar{k}\left(T_{0}\right) \leq \frac{1}{\sigma} \log \frac{K}{S_{0}}$ the option ceases to exist.

REMARK 4.1. It can be further proved that $\underline{k}\left(T_{0}\right)>-\infty$ is equivalent to the condition $T<\frac{\alpha}{1-\alpha} T_{0}$ and $\bar{k}\left(T_{0}\right)<\infty$ is equivalent to $T<\frac{1-\alpha}{\alpha} T_{0}$ respectively.

To describe the behaviour of the option's price let us denote for a moment the starting point of the option by $t_{0}$ and its total lifetime by $T_{1}=T_{0}+T$. Then $t\left(T_{0}, k\right)$ can be rewritten by

$$
t\left(T_{0}, k\right)=(1-\alpha) T_{1}-\int_{\tau=t_{0}}^{t_{0}+T_{0}} \mathbf{1}\left[Z_{\tau}>k\right] d \tau .
$$

Then $t\left(T_{0}, k\right)$ is decreasing for $T_{0} \rightarrow T_{1}$, i.e. $T \rightarrow 0$. Thus $\underline{k}\left(T_{0}\right)$ is increasing in $T_{0}$ and $\bar{k}\left(T_{0}\right)$ decreases since $t\left(T_{0}, k\right)>T$ is equivalent to

$$
T_{0}-\int_{\tau=t_{0}}^{t_{0}+T_{0}} \mathbf{1}\left[Z_{\tau}>k\right] d \tau>\alpha T_{1} .
$$

Obviously for $T_{0}=T_{1}$ we have $\underline{k}\left(T_{1}\right)=\bar{k}\left(T_{1}\right)$ and $F_{\alpha}$ is concentrated at this point, the $\alpha$-quantile of $Z$ in the time-period $\left[t_{0} ; t_{0}+T_{1}\right]$.

Remark 4.2. For $T_{0}=0$ we have $\underline{k}(0)=-\infty$ and $\bar{k}(0)=\infty$. Thus the option's price is 


$$
\pi_{B S}=r^{-T} S_{0} \sigma \int_{k>\frac{1}{\sigma} \log \frac{K}{S_{0}}}\left(1-F_{\alpha}(k)\right) \exp \{\sigma k\} d k
$$

with

$$
\begin{aligned}
F_{\alpha}(k) & =P\left[\Gamma_{+}(T, k)(Z) \leq(1-\alpha) T\right] \\
& = \begin{cases}1-F\left(T, \alpha T,-k ;-\frac{\mu_{B S}}{\sigma}\right), & k \geq 0 ; \\
F\left(T,(1-\alpha) T, k ; \frac{\mu_{B S}}{\sigma}\right), & k<0 .\end{cases}
\end{aligned}
$$

Now for values $\kappa<0$ we introduce the function $I$ by

$$
I(\tau, \theta, \kappa ; \nu, \sigma)=\int_{s=-\infty}^{\kappa} F(\tau, \theta, s ; \mu) \exp \{\sigma s\} d s .
$$

Using Lemma A.3 I can be calculated explicitly. We obtain the following representation,

$$
\begin{array}{r}
I(\tau, \theta, \kappa ; \mu, \sigma)= \\
\left\{\frac{2 \mu \kappa+2 \mu^{2} \tau+3}{2 \mu+\sigma}-\frac{2 \mu}{(2 \mu+\sigma)^{2}}\right\} \exp \{(2 \mu+\sigma) \kappa\} \mathcal{N}\left(\frac{\kappa}{\sqrt{\tau}}+\mu \sqrt{\tau},-\mu \sqrt{\tau-\theta} ;-\sqrt{1-\frac{\theta}{\tau}}\right) \\
-\frac{4(\mu+\sigma)^{2}}{\sigma(2 \mu+\sigma)^{2}} \exp \left\{(2 \mu+\sigma) \frac{\sigma}{2} \tau\right\} \mathcal{N}\left(\frac{\kappa}{\sqrt{\tau}}-(\mu+\sigma) \sqrt{\tau},(\mu+\sigma) \sqrt{\tau-\theta} ;-\sqrt{1-\frac{\theta}{\tau}}\right) \\
-\frac{4 \mu(\mu+\sigma)}{\sigma(2 \mu+\sigma)^{2}} \exp \left\{(2 \mu+\sigma) \frac{\sigma}{2} \theta\right\} \mathcal{N}\left(\frac{\kappa}{\sqrt{\theta}}-(\mu+\sigma) \sqrt{\theta}\right) \mathcal{N}(-\mu \sqrt{\tau-\theta}) \\
-\left\{\frac{2 \mu \kappa+2 \mu^{2} \theta}{2 \mu+\sigma}+\frac{\sigma}{(2 \mu+\sigma)^{2}}\right\} \exp \{(2 \mu+\sigma) \kappa\} \mathcal{N}\left(\frac{\kappa}{\sqrt{\theta}}+\mu \sqrt{\theta}\right) \mathcal{N}(-\mu \sqrt{\tau-\theta}) \\
-\frac{2 \mu}{2 \mu+\sigma} \sqrt{\frac{\theta}{2 \pi}} \exp \left\{(2 \mu+\sigma) \frac{\sigma}{2} \theta\right\} \exp \left\{-\frac{1}{2}\left(\frac{\kappa}{\sqrt{\theta}}-(\mu+\sigma) \sqrt{\theta}\right)^{2}\right\} \mathcal{N}(-\mu \sqrt{\tau-\theta}) \\
+\frac{2 \mu}{2 \mu+\sigma} \sqrt{\frac{\tau}{2 \pi}} \exp \left\{(2 \mu+\sigma) \frac{\sigma}{2} \tau\right\} \exp \left\{-\frac{1}{2}\left(\frac{\kappa}{\sqrt{\tau}}-(\mu+\sigma) \sqrt{\tau}\right)^{2}\right\} \mathcal{N}\left(\kappa \sqrt{\frac{1}{\theta}-\frac{1}{\tau}}\right) \\
+\frac{1}{\sigma} \exp \{\sigma \kappa\} \mathcal{N}\left(\frac{\kappa}{\sqrt{\tau}}-\mu \sqrt{\tau}, \mu \sqrt{\tau-\theta} ;-\sqrt{1-\frac{\theta}{\tau}}\right) \\
+\frac{2 \mu}{2 \mu+\sigma} \sqrt{\frac{\tau-\theta}{2 \pi}} \exp \left\{-\frac{\mu^{2}}{2}(\tau-\theta)\right\} \exp \{\sigma \kappa\} \mathcal{N}\left(\frac{\kappa}{\sqrt{\theta}}-\mu \sqrt{\theta}\right) \mathcal{N}(-\mu \sqrt{\tau-\theta})
\end{array}
$$


Then for $S_{0} \leq K$ the Black \& Scholes price of the option is determined by

$$
\pi_{B S}\left(\Phi_{0}\right)=r^{-T} S_{0} \sigma I\left(T, \alpha T, \frac{1}{\sigma} \log \frac{S_{0}}{K} ;-\frac{\mu_{B S}}{\sigma},-\sigma\right),
$$

and for $S_{0}>K$ by

$$
\begin{aligned}
& \pi_{B S}\left(\Phi_{0}\right)=r^{-T}\left(S_{0}-K\right)+r^{-T} S_{0} \sigma I\left(T,(1-\alpha) T, \frac{1}{\sigma} \log \frac{K}{S_{0}} ; \frac{\mu_{B S}}{\sigma}, \sigma\right) \\
& +r^{-T} S_{0} \sigma\left\{I\left(T, \alpha T, 0 ;-\frac{\mu_{B S}}{\sigma},-\sigma\right)-I\left(T,(1-\alpha) T, 0 ; \frac{\mu_{B S}}{\sigma}, \sigma\right)\right\} .
\end{aligned}
$$

Remark 4.3. Though Akahori (1995) and Dassios (1995) consider the Black \& Scholes price of an $\alpha$-quantile option they do not provide an explicit solution which can be exploited successfully for option pricing in practice. Especially when dealing with path-dependent options well-known approximative methods, e.g. numerical integration, Monte Carlo simulation or lattice approximation, often consume enormous resources with respect to accuracy and computation time whereas explicit formulae - if available - provide reliable prices nearly real time.

\section{CONCLUSION}

The main results of this paper are explicit representations for the density and the distribution of occupation times of Brownian motion with drift. By application of these distribution formulae to types of derivative securities where the payout depends on a certain amount of time-units exceeding a given boundary - e.g. quantile options are in this class of derivative securities - closed form solutions for the corresponding Black \& Scholes prices can be obtained which are appropriate for direct implementation in daily option trading.

REMARK 5.1. The author of this paper was motivated to his investigations in 1994 by the problem of an explicit pricing formula for a median option, i.e. a quantile option with quantile $\alpha=\frac{1}{2}$, independently from the research of Akahori and Dassios. He presented his results, i.e. Theorem 1.1 and Theorem 3.1 of this paper, at the conference "Derivatives '96", produced by RISK Publications, Geneva, 25 and 26 January, 1996.

\section{Appendix}

\section{A.1. Auxiliary Results}

In this appendix we provide some integration details for certain relations in the previous sections and auxiliary results used in this paper. 
A.1. Miscellaneous comments to Section 1

In the following some additional information concerning the results of Akahori (1995) is presented.

REMARK A.1. A straightforward derivation of Akahori's Theorem 1.1(i), i.e. eq. (3), is pointed out in detail. Using eq. (2) and Girsanov's theorem we obtain by Fubini's theorem

$$
\begin{aligned}
& P\left[\Gamma_{+}(T, 0)(Z) \in d u\right]=\exp \left\{-\frac{\nu^{2}}{2} T\right\} \int_{x \in g R} \exp \{\nu x\} P\left[\Gamma_{+}(T, 0)(W) \in d u ; W_{T} \in d x\right] \\
= & \frac{1}{2 \pi} \exp \left\{-\frac{\nu^{2}}{2} T\right\}\left\{\int_{t=u}^{T} \frac{d t}{t^{\frac{3}{2}}(T-t)^{\frac{1}{2}}}+\int_{t=T-u}^{T} \frac{d t}{t^{\frac{3}{2}}(T-t)^{\frac{1}{2}}}\right\} d u \\
& +\frac{\nu}{\sqrt{2 \pi}}\left\{\int_{t=u}^{T} \exp \left\{-\frac{\nu^{2}}{2} t\right\} \mathcal{N}(\nu \sqrt{T-t}) \frac{d t}{t^{\frac{3}{2}}}+\int_{t=T-u}^{T} \exp \left\{-\frac{\nu^{2}}{2} t\right\} \mathcal{N}(\nu \sqrt{T-t}) \frac{d t}{t^{\frac{3}{2}}}\right\} d u \\
& +\frac{2 \nu}{\sqrt{2 \pi T}} \exp \left\{-\frac{\nu^{2}}{2} T\right\} d u-\frac{2 \nu}{\sqrt{2 \pi u}} \exp \left\{-\frac{\nu^{2}}{2} u\right\} d u+2 \nu^{2} \mathcal{N}(\nu \sqrt{T}) d u-2 \nu^{2} \mathcal{N}(\nu \sqrt{u}) d u .
\end{aligned}
$$

By $\frac{T}{2} \int_{t=r}^{T} \frac{d t}{t^{\frac{3}{2}}(T-t)^{\frac{1}{2}}}=\sqrt{\frac{T-r}{r}}$ we obtain

$\frac{1}{2 \pi} \exp \left\{-\frac{\nu^{2}}{2} T\right\}\left\{\int_{t=u}^{T} \frac{d t}{t^{\frac{3}{2}}(T-t)^{\frac{1}{2}}}+\int_{t=T-u}^{T} \frac{d t}{t^{\frac{3}{2}}(T-t)^{\frac{1}{2}}}\right\} d u=\frac{1}{\pi} \exp \left\{-\frac{\nu^{2}}{2} T\right\} \frac{d u}{\sqrt{u(T-u)}}$.

By integration by parts we obtain small

$$
\begin{aligned}
& \frac{\nu}{\sqrt{2 \pi}} \int_{t=r}^{T} \exp \left\{-\frac{\nu^{2}}{2} t\right\} \mathcal{N}(\nu \sqrt{T-t}) \frac{d t}{t^{\frac{3}{2}}}=\left[\frac{-2 \nu}{\sqrt{2 \pi t}} \exp \left\{-\frac{\nu^{2}}{2} t\right\} \mathcal{N}(\nu \sqrt{T-t})\right]_{t=r}^{T} \\
& -\frac{\nu^{3}}{\sqrt{2 \pi}} \int_{t=r}^{T} \exp \left\{-\frac{\nu^{2}}{2} t\right\} \mathcal{N}(\nu \sqrt{T-t}) \frac{d t}{t^{\frac{1}{2}}}-\frac{\nu^{2}}{2 \pi} \exp \left\{-\frac{\nu^{2}}{2} T\right\} \int_{t=r}^{T} \frac{d t}{\sqrt{t(T-t)}} .
\end{aligned}
$$

Then we use the relations

$$
\begin{aligned}
& \int_{t=T-u}^{T} \frac{d t}{\sqrt{t(T-t)}}=\int_{t=0}^{u} \frac{d t}{\sqrt{t(T-t)}}, \\
& -\frac{\nu^{3}}{\sqrt{2 \pi}} \int_{t=T-u}^{T} \exp \left\{-\frac{\nu^{2}}{2} t\right\} \mathcal{N}(\nu \sqrt{T-t}) \frac{d t}{t^{\frac{1}{2}}}= \\
& -\frac{\nu^{3}}{\sqrt{2 \pi}} \int_{t=0}^{u} \exp \left\{-\frac{\nu^{2}}{2} t\right\} \mathcal{N}(\nu \sqrt{T-t}) \frac{d t}{t^{\frac{1}{2}}} \\
& +2 \nu^{2} \mathcal{N}(\nu \sqrt{T-u}) \mathcal{N}(\nu \sqrt{u})-\nu^{2} \mathcal{N}(\nu \sqrt{T})
\end{aligned}
$$


and the property

$$
\frac{\nu^{3}}{\sqrt{2 \pi}} \int_{t=0}^{T} \exp \left\{-\frac{\nu^{2}}{2} t\right\} \mathcal{N}(\nu \sqrt{T-t}) \frac{d t}{t^{\frac{1}{2}}}=\nu^{2} \mathcal{N}(\nu \sqrt{T})-\frac{\nu^{2}}{2} \exp \left\{-\frac{\nu^{2}}{2} T\right\}
$$

to conclude the final expression

$$
\begin{aligned}
P\left[\Gamma_{+}(T, 0)(Z) \in d u\right]= & \left\{\frac{1}{\pi} \exp \left\{-\frac{\nu^{2}}{2} T\right\} \frac{1}{\sqrt{u(T-u)}}+\sqrt{\frac{2}{\pi}} \frac{\nu}{\sqrt{T-u}} \exp \left\{-\frac{\nu^{2}}{2}(T-u)\right\} \mathcal{N}(\nu \sqrt{u})\right. \\
& \left.-\sqrt{\frac{2}{\pi}} \frac{\nu}{\sqrt{u}} \exp \left\{-\frac{\nu^{2}}{2} u\right\} \mathcal{N}(-\nu \sqrt{T-u})-2 \nu^{2} \mathcal{N}(\nu \sqrt{u}) \mathcal{N}(-\nu \sqrt{T-u})\right\} d u .
\end{aligned}
$$

REMARK A.2. It can be shown that the representation of $F(\tau, \theta, \kappa ; \mu)$ for $\kappa<0$ in Theorem 1.1 holds for $\kappa>0$, respectively.

\section{A.2. Miscellaneous comments to Sections 3 and 4}

In Section 3 we omitted the technical proof of Theorem 3.1. In the following we provide the ideas behind the explicit computations of all partial derivatives of the function $D$ introduced by eq. (9). As mentioned before the distribution of occupation times can be represented completely by a linear combination of those partial derivatives.

Proof of Theorem 3.1. For $\kappa<0$ we know the following representation for $F$ by Theorem 1.1,

$$
\begin{aligned}
F(\tau, \theta, \kappa ; \mu)= & -2 \mu^{2} \exp \{2 \kappa \mu\} D(\tau, \theta, \kappa ; \mu)-2 \mu \exp \{2 \kappa \mu\} \frac{\partial}{\partial \kappa} D(\tau, \theta, \kappa ; \mu) \\
& -4 \exp \{2 \kappa \mu\} \frac{\partial}{\partial \tau} D(\tau, \theta, \kappa ; \mu)-\frac{4}{\mu} \exp \{2 \kappa \mu\} \frac{\partial^{2}}{\partial \tau \partial \kappa} D(\tau, \theta, \kappa ; \mu)
\end{aligned}
$$

Furthermore the corresponding partial derivatives of $d(\tau, \theta, \kappa ; \mu)=\frac{\partial}{\partial \theta} D(\tau, \theta, \kappa ; \mu)$ are known by Remark 1.3. We start with the evaluation of $\frac{\partial^{2}}{\partial \tau \partial \kappa} D(\tau, \theta, \kappa ; \mu)$ and calculate the integral

$H(\kappa):=\frac{1}{\pi} \int_{s=0}^{\theta} \frac{1}{\sqrt{\tau-s} \sqrt{s}} \exp \left\{-\frac{\kappa^{2}}{2 s}\right\} d s=\frac{2}{\pi} \int_{w=\sqrt{\frac{\tau}{\theta}-1}}^{\infty} \frac{1}{w^{2}+1} \exp \left\{-\frac{\kappa^{2}}{2 \tau}\left(w^{2}+1\right)\right\} d w$.

By differentiation of this integral representation to $\kappa$ we obtain

$$
H^{\prime}(\kappa)=2 \sqrt{\frac{2}{\pi \tau}} \exp \left\{-\frac{\kappa^{2}}{2 \tau}\right\} \mathcal{N}\left(\kappa \sqrt{\frac{1}{\theta}-\frac{1}{\tau}}\right)
$$


and by Lemma A.3, eq. (A9), $H(\kappa)=4 \mathcal{N}\left(\frac{\kappa}{\sqrt{\tau}}, 0 ;-\sqrt{1-\frac{\theta}{\tau}}\right)$. Thus we have

$$
\frac{\partial^{2}}{\partial \tau \partial \kappa} D(\tau, \theta, \kappa ; \mu)=-\mu \exp \left\{-\frac{\mu^{2}}{2} \tau\right\} \exp \{-\kappa \mu\} \mathcal{N}\left(\frac{\kappa}{\sqrt{\tau}}, 0 ;-\sqrt{1-\frac{\theta}{\tau}}\right) .
$$

In the next step we calculate $\frac{\partial}{\partial \tau} D(\tau, \theta, \kappa ; \mu)$ by integration with respect to $\kappa$ applying Lemma A.3, eq. (A4). We obtain explicitly

$$
\begin{aligned}
\frac{\partial}{\partial \tau} D(\tau, \theta, \kappa ; \mu)= & \exp \left\{-\frac{\mu^{2}}{2} \tau\right\} \exp \{-\kappa \mu\} \mathcal{N}\left(\frac{\kappa}{\sqrt{\tau}}, 0 ;-\sqrt{1-\frac{\theta}{\tau}}\right) \\
& -\mathcal{N}\left(\frac{\kappa}{\sqrt{\tau}}+\mu \sqrt{\tau},-\mu \sqrt{\tau-\theta} ;-\sqrt{1-\frac{\theta}{\tau}}\right) .
\end{aligned}
$$

Similarly to the crucial idea in our proof of Dassios' identity in law we use integration by parts for the computation of $\frac{\partial}{\partial \kappa} D(\tau, \theta, \kappa ; \mu)$ to avoid integration with respect to $\tau$ which appears to be somewhat complicated. We obtain the following relation which shows the interesting relation between $\frac{\partial}{\partial \kappa} D(\tau, \theta, \kappa ; \mu)$ and $\frac{\partial}{\partial \tau} D(\tau, \theta, \kappa ; \mu)$

$$
\begin{aligned}
\frac{\partial}{\partial \kappa} D(\tau, \theta, \kappa ; \mu)= & \frac{1}{\mu}\left\{\mathcal{N}\left(\frac{\kappa}{\sqrt{\theta}}+\mu \sqrt{\theta}\right)+\right. \\
& \left.\exp \{-2 \kappa \mu\} \mathcal{N}\left(-\frac{\kappa}{\sqrt{\theta}}+\mu \sqrt{\theta}\right)\right\} \mathcal{N}(-\mu \sqrt{\tau-\theta}) \\
& +\frac{1}{\mu}\left\{\frac{\partial}{\partial \tau} D(\tau, \theta, \kappa ; \mu)+\exp \{-2 \kappa \mu\} \frac{\partial}{\partial \tau} D(\tau, \theta,-\kappa ; \mu)-\right. \\
& \exp \{-2 \kappa \mu\} \mathcal{N}(-\mu \sqrt{\tau})\} .
\end{aligned}
$$

By Lemma A.3, eq. (A4), $\frac{\partial}{\partial \tau} D(\tau, \theta,-\kappa ; \mu)$ can be calculated analogously to $\frac{\partial}{\partial \tau} D(\tau, \theta, \kappa ; \mu)$, i.e.

$$
\begin{aligned}
\frac{\partial}{\partial \tau} D(\tau, \theta,-\kappa ; \mu)= & \mathcal{N}(\mu \sqrt{\tau-\theta})-\mathcal{N}(\mu \sqrt{\tau})- \\
& \mathcal{N}\left(\frac{\kappa}{\sqrt{\theta}}-\mu \sqrt{\theta}, \mu \sqrt{\tau-\theta} ;-\sqrt{1-\frac{\theta}{\tau}}\right) \\
& +\exp \left\{-\frac{\mu^{2}}{2} \tau\right\} \exp \{\kappa \mu\} \mathcal{N}\left(\frac{\kappa}{\sqrt{\tau}}, 0 ;-\sqrt{1-\frac{\theta}{\tau}}\right) .
\end{aligned}
$$


Now we summarize all explicit terms of $\frac{\partial}{\partial \kappa} D(\tau, \theta, \kappa ; \mu)$ for the following explicit representation,

$$
\begin{aligned}
& \frac{\partial}{\partial \kappa} D(\tau, \theta, \kappa ; \mu)=\frac{1}{\mu}\left\{\mathcal{N}\left(\frac{\kappa}{\sqrt{\theta}}+\mu \sqrt{\theta}\right)-\right. \\
& \left.\exp \{-2 \kappa \mu\} \mathcal{N}\left(\frac{\kappa}{\sqrt{\theta}}-\mu \sqrt{\theta}\right)\right\} \mathcal{N}(-\mu \sqrt{\tau-\theta}) \\
& +\frac{2}{\mu} \exp \left\{-\frac{\mu^{2}}{2} \tau\right\} \exp \{-\kappa \mu\} \mathcal{N}\left(\frac{\kappa}{\sqrt{\tau}}, 0 ;-\sqrt{1-\frac{\theta}{\tau}}\right) \\
& -\frac{1}{\mu} \mathcal{N}\left(\frac{\kappa}{\sqrt{\tau}}+\mu \sqrt{\tau},-\mu \sqrt{\tau-\theta} ;-\sqrt{1-\frac{\theta}{\tau}}\right) \\
& -\frac{1}{\mu} \exp \{-2 \kappa \mu\} \mathcal{N}\left(\frac{\kappa}{\sqrt{\tau}}-\mu \sqrt{\tau}, \mu \sqrt{\tau-\theta} ;-\sqrt{1-\frac{\theta}{\tau}}\right),
\end{aligned}
$$

and by integration to $\kappa$ using Lemma A.3, eqs. (A1), (A3), (A4), we have

$$
\begin{aligned}
D(\tau, \theta, \kappa ; \mu)= & \left\{\frac{3}{2 \mu^{2}}-\frac{\kappa}{\mu}-\tau\right\} \mathcal{N}\left(\frac{\kappa}{\sqrt{\tau}}+\mu \sqrt{\tau},-\mu \sqrt{\tau-\theta} ;-\sqrt{1-\frac{\theta}{\tau}}\right) \\
& +\frac{1}{2 \mu^{2}} \exp \{-2 \kappa \mu\} \mathcal{N}\left(\frac{\kappa}{\sqrt{\tau}}-\mu \sqrt{\tau}, \mu \sqrt{\tau-\theta} ;-\sqrt{1-\frac{\theta}{\tau}}\right) \\
& -\frac{2}{\mu^{2}} \exp \left\{-\frac{\mu^{2}}{2} \tau\right\} \exp \{-\kappa \mu\} \mathcal{N}\left(\frac{\kappa}{\sqrt{\tau}}, 0 ;-\sqrt{1-\frac{\theta}{\tau}}\right) \\
& +\left\{\frac{\kappa}{\mu}-\frac{1}{2 \mu^{2}}+\theta\right\} \mathcal{N}\left(\frac{\kappa}{\sqrt{\theta}}+\mu \sqrt{\theta}\right) \mathcal{N}(-\mu \sqrt{\tau-\theta}) \\
& +\frac{1}{2 \mu^{2}} \exp \{-2 \kappa \mu\} \mathcal{N}\left(\frac{\kappa}{\sqrt{\theta}}-\mu \sqrt{\theta}\right) \mathcal{N}(-\mu \sqrt{\tau-\theta}) \\
& +\frac{1}{\mu} \sqrt{\frac{\theta}{2 \pi}} \exp \left\{-\frac{1}{2}\left(\frac{\kappa}{\sqrt{\theta}}+\mu \sqrt{\theta}\right)^{2}\right\} \mathcal{N}(-\mu \sqrt{\tau-\theta}) \\
& -\frac{1}{\mu} \sqrt{\frac{\tau}{2 \pi}} \exp \left\{-\frac{1}{2}\left(\frac{\kappa}{\sqrt{\tau}}+\mu \sqrt{\tau}\right)^{2}\right\} \mathcal{N}\left(\kappa \sqrt{\frac{1}{\theta}-\frac{1}{\tau}}\right) \\
& +\frac{1}{\mu} \sqrt{\frac{\tau-\theta}{2 \pi}} \exp \left\{-\frac{\mu^{2}}{2}(\tau-\theta)\right\} \mathcal{N}\left(\frac{\kappa}{\sqrt{\theta}}+\mu \sqrt{\theta}\right) .
\end{aligned}
$$

Finally we summarize those results and calculate $F(\tau, \theta, \kappa ; \mu)$ explicitly.

For the explicit calculation of integrals related to the normal distribution in Section 3 and Section 4 we referred essentially to the following lemma.

LEMMA A.3. The following integral relations hold. 
(A1) $\int \mathcal{N}(a x+b) d x=$

$\left(x+\frac{b}{a}\right) \mathcal{N}(a x+b)+\frac{1}{a \sqrt{2 \pi}} \exp \left\{-\frac{1}{2}(a x+b)^{2}\right\}$.

(A2)

$\int \exp \{c x\} \mathcal{N}(a x+b) d x=$
$\frac{1}{c} \exp \{c x\} \mathcal{N}(a x+b)-\frac{1}{c} \exp \left\{\frac{c^{2}}{2 a^{2}}-\frac{b c}{a}\right\} \mathcal{N}\left(a x+b-\frac{c}{a}\right)$.

(A3) $\int \mathcal{N}(a x+b, y ; \rho) d x=$

$\left(x+\frac{b}{a}\right) \mathcal{N}(a x+b, y ; \rho)$

$+\frac{1}{a \sqrt{2 \pi}} \exp \left\{-\frac{1}{2}(a x+b)^{2}\right\} \mathcal{N}\left(\frac{y-\rho(a x+b)}{\sqrt{1-\rho^{2}}}\right)$

$+\frac{\rho}{a \sqrt{2 \pi}} \exp \left\{-\frac{y^{2}}{2}\right\} \mathcal{N}\left(\frac{(a x+b)-\rho y}{\sqrt{1-\rho^{2}}}\right)$.

(A4) $\begin{aligned} & \int_{1} \exp \{c x\} \mathcal{N}(a x+b, y ; \rho) d x= \\ & \frac{1}{c} \exp \{c x\} \mathcal{N}(a x+b, y ; \rho)\end{aligned}$

$-\frac{1}{c} \exp \left\{\frac{c^{2}}{2 a^{2}}-\frac{b c}{a}\right\} \mathcal{N}\left(a x+b-\frac{c}{a}, y-\frac{c \rho}{a} ; \rho\right)$.

(A5) $\int x \mathcal{N}(a x+b) d x=$

$\left(\frac{x^{2}}{2}-\frac{b^{2}+1}{2 a^{2}}\right) \mathcal{N}(a x+b)+\frac{a x-b}{2 a^{2} \sqrt{2 \pi}} \exp \left\{-\frac{1}{2}(a x+b)^{2}\right\}$.

(A6) $\int x \exp \{c x\} \mathcal{N}(a x+b) d x=$

$$
\begin{aligned}
& \frac{c x-1}{c^{2}} \exp \{c x\} \mathcal{N}(a x+b) \\
& +\frac{a^{2}+a b c-c^{2}}{a^{2} c^{2}} \exp \left\{\frac{c^{2}}{2 a^{2}}-\frac{b c}{a}\right\} \mathcal{N}\left(a x+b-\frac{c}{a}\right) \\
& +\frac{1}{a c \sqrt{2 \pi}} \exp \left\{\frac{c^{2}}{2 a^{2}}-\frac{b c}{a}\right\} \exp \left\{-\frac{1}{2}\left(a x+b-\frac{c}{a}\right)^{2}\right\} .
\end{aligned}
$$


(A7)

$$
\begin{aligned}
& \int x \mathcal{N}(a x+b, y ; \rho) d x= \\
& \left(\frac{x^{2}}{2}-\frac{b^{2}+1}{2 a^{2}}\right) \mathcal{N}(a x+b, y ; \rho) \\
& +\frac{a x-b}{2 a^{2} \sqrt{2 \pi}} \exp \left\{-\frac{1}{2}(a x+b)^{2}\right\} \mathcal{N}\left(\frac{y-\rho(a x+b)}{\sqrt{1-\rho^{2}}}\right) \\
& -\frac{\rho(2 b-\rho y)}{2 a^{2} \sqrt{2 \pi}} \exp \left\{-\frac{y^{2}}{2}\right\} \mathcal{N}\left(\frac{(a x+b)-\rho y}{\sqrt{1-\rho^{2}}}\right) \\
& -\frac{\rho \sqrt{1-\rho^{2}}}{4 a^{2} \pi} \exp \left\{-\frac{y^{2}}{2}\right\} \exp \left\{\begin{array}{c}
\left.-\frac{(a x+b-\rho y)^{2}}{2\left(1-\rho^{2}\right)}\right\}
\end{array}\right.
\end{aligned}
$$

(A8) $\int x \exp \{c x\} \mathcal{N}(a x+b, y ; \rho) d x=$ $\left(\frac{x}{c}-\frac{1}{c^{2}}\right) \exp \{c x\} \mathcal{N}(a x+b, y ; \rho)$ $+\frac{a^{2}+a b c-c^{2}}{a^{2} c^{2}} \exp \left\{\frac{c^{2}}{2 a^{2}}-\frac{b c}{a}\right\} \mathcal{N}\left(a x+b-\frac{c}{a}, y-\frac{c}{a} \rho ; \rho\right)$ $+\frac{1}{a c \sqrt{2 \pi}} \exp \{c x\} \exp \left\{-\frac{1}{2}(a x+b)^{2}\right\} \mathcal{N}\left(\frac{y-\rho(a x+b)}{\sqrt{1-\rho^{2}}}\right)$ $+\frac{\rho}{a c \sqrt{2 \pi}} \exp \left\{\frac{c^{2}-2 a b c-(a y-c \rho)^{2}}{2 a^{2}}\right\}$ $\times \mathcal{N}\left(\frac{a x+b-\rho y}{\sqrt{1-\rho^{2}}}-\frac{c}{a} \sqrt{1-\rho^{2}}\right)$.

With respect to bivariate normal distribution functions the following relation may be sometimes helpful. For all $x, y, p \in g R$ we have

$$
\mathcal{N}\left(x, \frac{y}{\sqrt{1+p^{2}}} ;-\frac{p}{\sqrt{1+p^{2}}}\right)=\frac{1}{\sqrt{2 \pi}} \int_{t=-\infty}^{x} \exp \left\{-\frac{t^{2}}{2}\right\} \mathcal{N}(y+p t) d t
$$

Proof. Lemma A.3 can be easily proved by differentiation with respect to $x$.

\section{References}

1. Akahori, J. (1995). "Some Formulae for a New Type of Path-Dependent Option." Ann. Appl. Probab. 5 383-388.

2. Billingsley, P. (1968). Convergence of Probability Measures. Wiley, New York.

3. DAssios, A. (1995). "The Distribution of the Quantiles of a Brownian Motion with Drift and the Pricing of Path-Dependent Options." Ann. Appl. Probab. 5 389-398.

4. Duffie, D. (1988). Security Markets - Stochastic Models. Academic Press, New York.

5. Embrechts, P., Rogers, L. C. G. and Yor, M. (1995). "A Proof of Dassios' Representation of the $\alpha$-Quantile of Brownian Motion with Drift." Ann. Appl. Probab. 5 757-767. 
6. Harrison, J. M. and Pliska, S. R. (1981). "Martingales and Stochastic Integrals in the Theory of Continuous Trading." Stochastic Process. Appl. 15 214-260.

7. Karatzas, I. and Shreve, S. E. (1984). "Trivariate Density of Brownian Motion, its Local and Occupation Times, with Application to Stochastic Control." Ann. Probab. 12 819-828.

8. Karatzas, I. and Shreve, S. E. (1988). Brownian Motion and Stochastic Calculus. Springer, Berlin. 


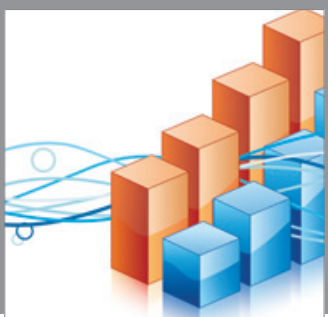

Advances in

Operations Research

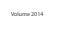

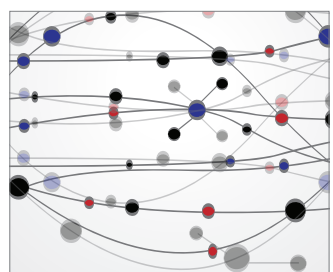

\section{The Scientific} World Journal
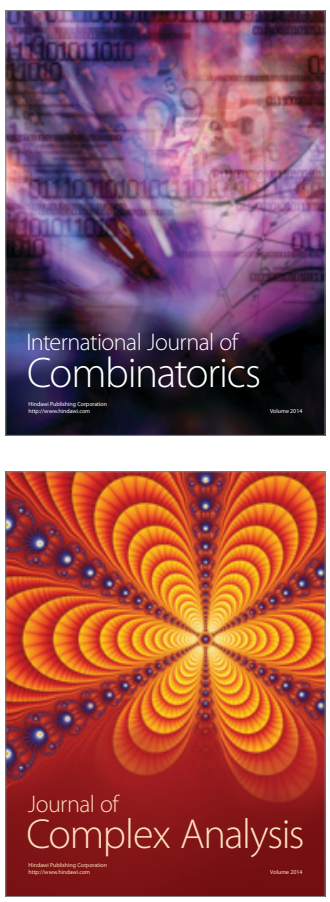

International Journal of

Mathematics and

Mathematical

Sciences
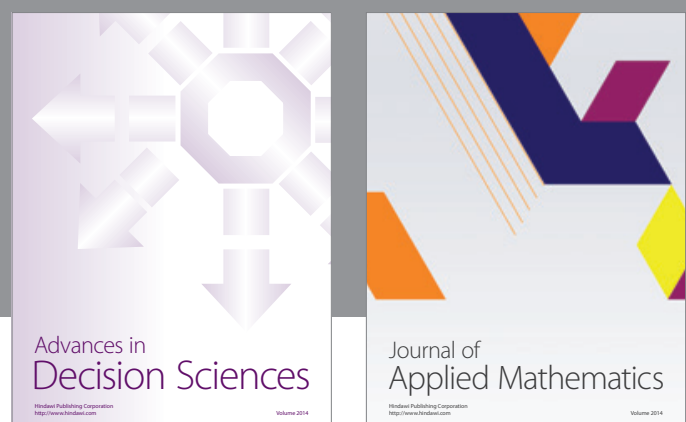

Journal of

Applied Mathematics
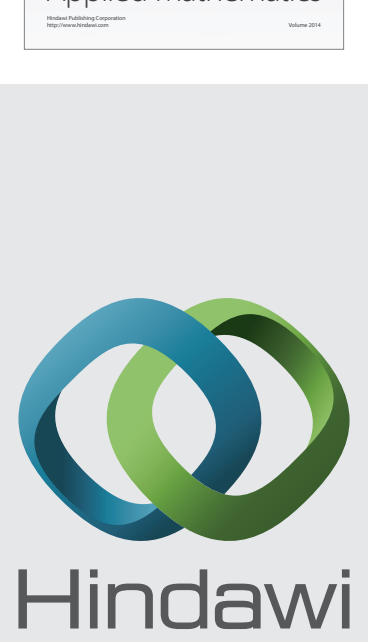

Submit your manuscripts at http://www.hindawi.com
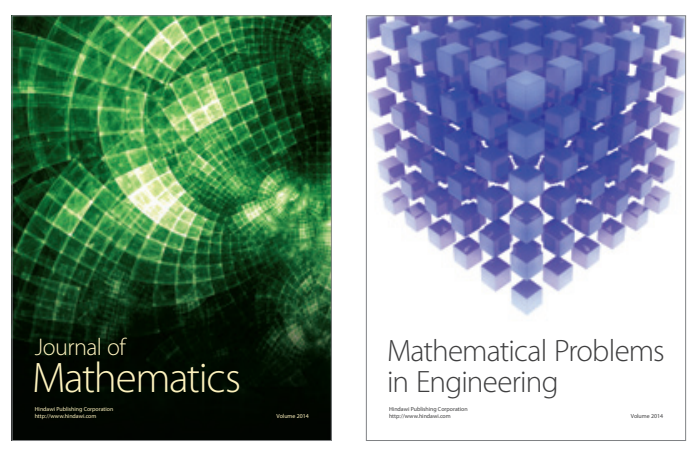

Mathematical Problems in Engineering
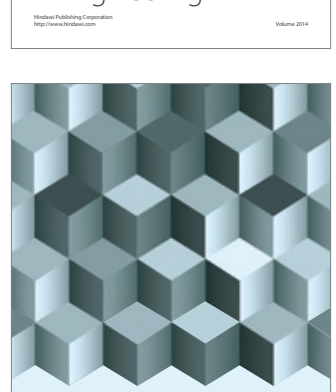

Journal of

Function Spaces
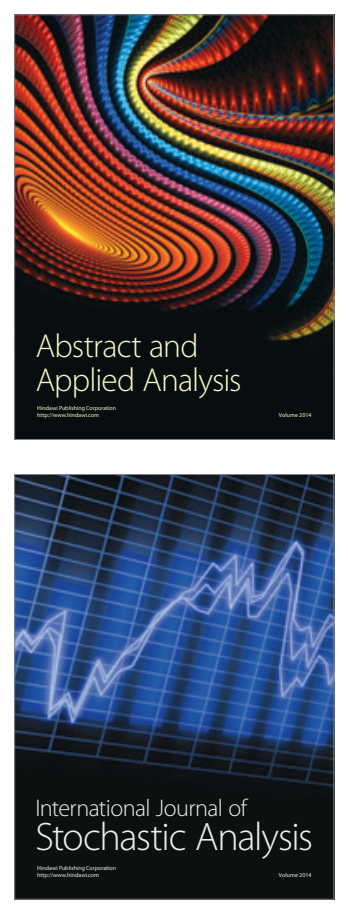

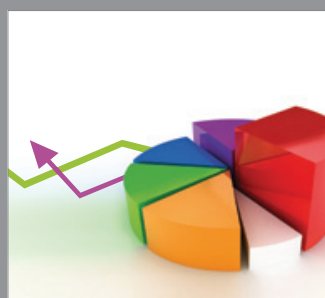

ournal of

Probability and Statistics

Promensencen
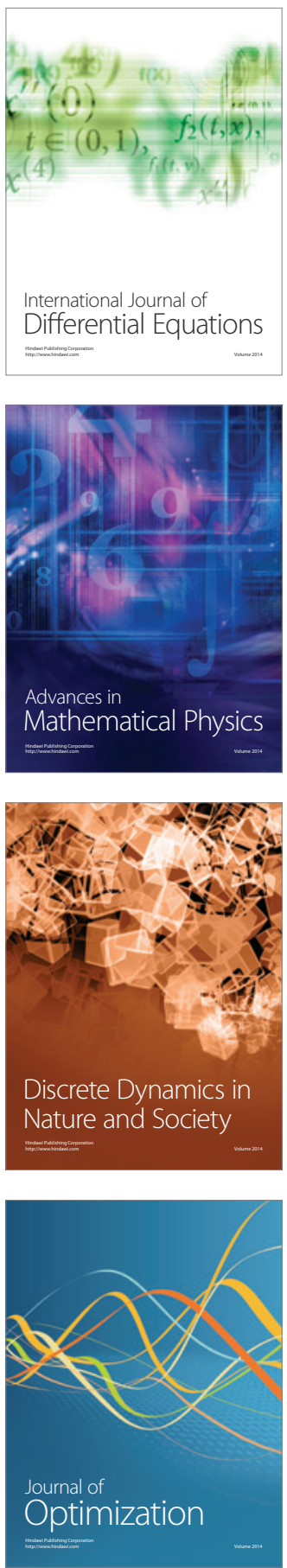